\title{
A Lesser Known Stroke Mimic: Posterior Reversible Encephalopathy Syndrome
}

\author{
Keneilwe Malomo ${ }^{1}$, Ontefetse Ntlholang ${ }^{2}$ \\ ${ }^{1}$ Department of medicine for the elderly, Connolly Hospital, Blanchardstown, Ireland \\ ${ }^{2}$ Medicine for the elderly directorate, St James Hospital, Dublin 8, Ireland
}

Received: 04/01/2016

Accepted: $17 / 01 / 2016$

Published: $29 / 02 / 2016$

How to cite this article: Malomo K, Ntlholang O. A lesser known stroke mimic: posterior reversible encephalopathy syndrome. EJCRIM 2016;3:doi:10.12890/2016_000376

Conflicts of Interests: The authors declare that there are no competing interests.

This article is licensed under a Commons Attribution Non-Commercial 4.0 License

\section{ABSTRACT}

Posterior reversible encephalopathy syndrome (PRES) is a clinico-neuro-radiological diagnosis, which can complicate a wide range of conditions. Clinical features include generalised and/or focal neurological deficits. These features are also present in neurovascular disorders, such as stroke. Currently, emphasis in the management of hyperacute stroke is thrombolysis, and it is important to bear in mind stroke mimics as a possible cause of clinical features. The Authors present the case of a 66-year-old man, who presented with acute focal neurological deficit. His brain imaging and history were consistent with PRES.

\section{LEARNING POINTS}

- Posterior reversible encephalopathy syndrome (PRES) should be considered as a possibility in hyperacute stroke, especially those with posterior cerebral symptoms and signs.

- Serial CT brain scanning may be helpful when MRI is unavailable or contraindicated.

- Early recognition and management of this syndrome would prevent permanent brain damage and unnecessary investigations and treatments.

\section{KEYWORDS}

Posterior reversible encephalopathy syndrome; hyperacute stroke; stroke mimics; thrombolysis; brain imaging.

\section{INTRODUCTION}

Posterior reversible encephalopathy syndrome (PRES) is a clinico-neuro-radiological diagnosis complicating a wide range of conditions, first described by Hinchey et al[ ${ }^{[1]}$. Clinical features include headache, vomiting, altered sensation, disturbances of vision, seizures and focal deficits $^{[2]}$. Radiologically, there is predominant posterior leukoencephalopathy with vasogenic oedema ${ }^{[3]}$.

\section{CASE PRESENTATION}

A 66-year-old man with a background history of subdural haematoma, ankylosing spondylitis, autonomic dysfunction, permanent pacemaker, ischaemic heart disease, and essential tremor presented to Emergency Department following a fall. He felt a strange feeling all over the body and fell. He had no recollection until about 4 hours later, when he heard the telephone ring. When he tried to move, he noticed leftsided weakness. His medications included rosuvastatin, venlafaxine, fludrocortisone, and midodrine. 


\section{European Journal}

of Case Reports in

Internal Medicine

Examination revealed laceration on his right scalp; blood pressure 155/99mmHg; heart rate 72 beats-per-minute; Glasgow Coma Scale 15/15; right facial droop; and left arm drift. He was FAST (Face, Arm, Speech, Time) positive, with ROSIER score of 2 and NIHSS score of 7/42. He would have been treated with thrombolysis had it not been for the computed tomography (CT) findings. Two days later, he had left-visual and hemi-body neglect, right-facial droop, left-arm drift, and limbs dyspraxia. Seven days post-admission, his neurological examination revealed marked improvement.

Non-contrast computed tomography (NCCT) brain scan revealed deep white matter changes in both occipital lobes, with effacement of the sulci over the vertex in keeping with cerebral oedema (Fig. 1). CT brain scan, with contrast on day 2 , showed low attenuation changes in the right cerebellar peduncle and white matter of both occipital poles and posterior parietal lobes (Fig. 2). Repeat NCCT on day 5 showed increased cerebral oedema (Fig. 3). NCCT brain scan on day 13 showed less extensive symmetrical low densities (Fig. 4).

The patient was diagnosed with PRES secondary to severe autonomic dysfunction, with midodrine as a possible contributor. Other differential diagnoses on presentation were metabolic derangement (prolonged hypoglycaemia) and acute cerebral ischaemia. As part of management, midodrine was stopped. He underwent extensive rehabilitation before being discharged from hospital.
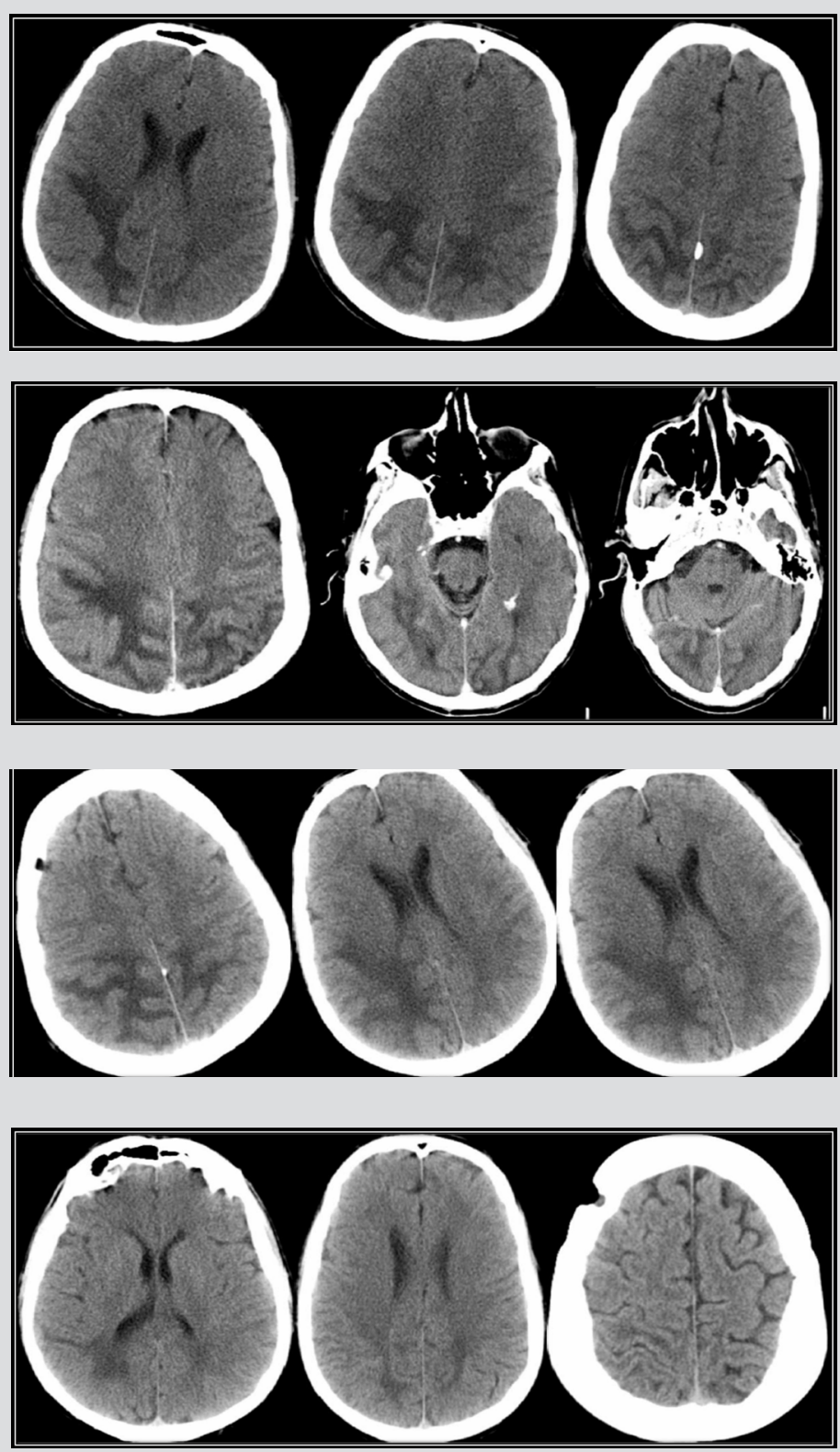

Figure 1: CT brain scan on day 1 - Deep white matter change in both occipital lobes slightly more marked on the right side, with effacement of the sulci over the vertex in keeping with cerebral oedema.

Figure 2: CT brain scan with contrast on day 2 - Low attenuation changes in the right cerebellar peduncle and white matter of both occipital poles and posterior parietal lobes.

Figure 3: CT brain scan on day 5 - Increased cerebral oedema.

Figure 4: CT brain scan on day 13 - Less extensive symmetrical low densities. 


\section{DISCUSSION}

PRES is well described in literature, but it is a lesser known stroke mimic presenting with acute focal neurological deficits. Exogenous factors like drugs have been implicated in the development of PRES; among them is midodrine, a selective $\alpha-1$ adrenoreceptor ${ }^{[4]}$.

Our patient did not have a magnetic resonance imaging (MRI) brain scan due to a permanent pacemaker; instead we ran serial computed tomographies (CT). On patients who had CT and MRI brain scans, only $32 \%$ and $74 \%$ had contributory CT findings on day 1 and 2 , respectively, suggesting that repeated CT scanning may be helpful when MRI is unavailable or contraindicated ${ }^{[5]}$. MRI is superior to $\mathrm{CT}$ for the diagnosis of PRES. Occipital, parietal, frontal, temporal in decreasing order of frequency, and other parts of the brain can be affected ${ }^{[5]}$. These will lead to different clinical presentation depending on the area(s) affected.

With the advent of stroke thrombolysis, these patients are more likely to be thrombolysed with normal CT brain scan findings. A high index of suspicion is needed from history and clinical examination. Moreover, early recognition and timely management of this syndrome is important as it prevents permanent brain damage and unnecessary investigations and treatments.

\section{REFERENCES}

1. Hinchey J, Chaves C, Appignani B, Breen J, Pao L, Wang A, et al. A reversible posterior leukoencephalopathy syndrome. N Engl J Med 1996;334:494-500.

2. Legriel S, Pico F, Azoulay E. Understanding Posterior Reversible Encephalopathy Syndrome. J.-L. Vincent (ed.), Annual Update in Intensive Care and Emergency Medicine 2011.

3. Pasupuleti DV, Miranda M, Vattipally V. Case Report: Posterior Reversible Encephalopathy Syndrome. Am Fam Physician 2005;72:2430-2436.

4. Pedraza R, Marik PE, Varon J. Posterior Reversible Encephalopathy Syndrome: A Review. Crit Care \& Shock 2009;12:135-143.

5. Bartynski WS, Boardman JF. Distinct imaging patterns and lesion distribution in posterior reversible encephalopathy syndrome. AJNR Am J Neuroradiol 2007;28:1320-1327. 